\title{
EVALUATION OF MULTIMODAL THERAPY USING ENDOSCOPIC RESECTION WITH CONCURRENT GEMCITABINE AND RADIOTHERAPY IN THE TREATMENT OF MUSCLE-INVASIVE BLADDER CANCER
}

\author{
Abd El Rahman M. El Fayoumi, M.D.*; Ashraf S. Shahin, M.D.*; Talaat E. El Mokadem, M.D.**; Maged \\ M. Ali*; Abd El Motaleb Mohammed, M.D.** \\ * Urology department, ** Clinical oncology and Nuclear Medicine department, Faculty of Medicine, Zagazig \\ University.
}

Background: Radical cystectomy is the preferred line of treatment for MIBC. Multimodal therapy including concomitant chemo-radiotherapy gives promising results comparable to cystectomy especially in those patients with solitary, low stage, small size tumors that are amenable to complete resection.

Aim of the work: to evaluate the role of bladder preservation protocol including concomitant chemoradiotherapy in the treatment of muscle invasive bladder cancer as regard: local control, systemic control, and quality of life.

Materials and methods: Forty patients with primary (T2-4a, Nx, M0) TCC of the bladder with adequate performance status and eligible for cystectomy were enrolled over a period from March 2011 to July 2013. All patients are subjected to maximal TURBT, Intra-venous gemcitabine infusion $\left(300 \mathrm{mg} / \mathrm{m}^{2}\right.$ body surface area weekly during radiotherapy), and 60 Gy $/ 6$ weeks using linear accelerator 4-6 mev. $1^{\text {st }}$ phase:- total pelvic radiation of 40 Gy by dose of 20 fractions in 20 days. While $2^{\text {nd }}$ phase:- localized pelvic field dose of 20 Gy by 10 fractions in 10 days. The endpoint of the study is either treatment failure or completion of the follow up period via cystoscopy every three months for 2 years, and upper tract surveillance was performed by ultrasonography every six months and CT yearly.

Results: After phase I; 22 (55\%) patients had complete response (complete disappearance of all measurable lesions), 8 (20\%) patients had partial response (more than 50\% reduction of all measurable lesions or no increase in any lesion or no new lesions) and 10 (25\%) patients had stable disease (less than 50\% reduction of measurable lesions or $<25 \%$ increase of measurable lesions). Patients who had stable disease were subjected to salvage cystectomy. Patients who had CR and PR (totally 30 patients) started phase II. After phase II, only 3 patients underwent salvage chemotherapy and 27 patients had CR. At the end of treatment protocol, 27 (67.5\%) patients had CR with bladder preservation while $13(32.5 \%)$ patients were subjected to salvage cystectomy. KaplanMeier analysis of recurrence-free survival, overall survival of the patients was done.

Conclusion: Bladder preservation protocol including concomitant chemo-radiotherapy gives comparable results with cystectomy in local control of the disease and survival rates of the patients with better quality of life especially in those patients with solitary $\mathrm{T} 2$, high grade tumors that are less than $5 \mathrm{~cm}$ and are amenable to complete resection.

\section{INTRODUCTION}

B ladder carcinoma is the second most common malignant neoplasm of the genitourinary tract. In men, it is the fourth most common cancer after prostate, lung, and colorectal cancer, accounting for $6.6 \%$ of all cancer cases. In women, it is the ninth most common cancer, accounting for $2.4 \%$ of all cancers diagnosed annually in United States increased by over $50 \%$, at a $25 \%$ faster rate in men than in women. Approximately $30 \%$ of the bladder tumors are diagnosed as muscle invasive tumors at first diagnosis. Muscle-invasive bladder cancer (MIBC) is a disease associated with a relatively low cure rate.

The optimal management of MIBC has been a continuous subject of controversy. Radical cystectomy represents the most frequent treatment approach. Despite great advances in surgical techniques and better perioperative support, even a neo-bladder cannot substitute for the patient's original bladder without entailing a high risk of infection and consequential renal failure, metabolic disorders, and sexual dysfunction. Another drawback is that approximately half of the patients will die with distant metastases despite cystectomy. For bilharzial bladder cancer, local recurrence represented $50 \%-60 \%$ of the causes of failure after radical cystectomy.

Bladder preservation with aggressive transurethral surgery, systemic chemotherapy, and radiotherapy has resulted in 5-year survival rates approximately equivalent to those after cystectomy (60\%), with $40 \%$ of patients surviving with an intact bladder. It has been assumed that patients with a 
preserved bladder have improved quality of life (QOL) compared with patients who undergo radical cystectomy.

Multimodal therapy including concomitant chemo-radiotherapy gives promising results comparable to cystectomy in local control of the disease and survival rates of the patients with better quality of life specially in those patients with solitary T2, low grade tumors that are less than $5 \mathrm{~cm}$ and are amenable to complete resection.

\section{AIM OF THE WORK}

To evaluate the role of bladder preservation protocol including concomitant chemo-radiotherapy in the treatment of muscle invasive bladder cancer as regard: local control, systemic control, and quality of life.

\section{PATIENTS AND METHODS}

This prospective study was carried out at the Department of Urology in collaboration with the department of Clinical Oncology and Nuclear Medicine, Zagazig University Hospitals between April 2011 and July 2013. The study included 40 patients diagnosed clinically and radiologicaly as (cT2-4a, Nx, M0) and the primary lesion of the bladder was confirmed to be $\mathrm{T} 2-4 \mathrm{a}$ in histopathological examination of the initial TURBT. Tumors were staged according to the 2009 TumorNode-Metastasis (TNM) classification of urinary bladder cancer. Tumors were graded according to the 2004 World Health Organization (WHO) grading of urinary tumors. All histopathological examinations of the resected tissues were performed at the Department of Pathology at our university. Informed consent regarding the treatment of bladder cancer was obtained from every patient.

Inclusion criteria: Primary T2-T4a, Nx, M0 Transitional cell carcinoma of the bladder. Serum creatinine level $1.5-2 \mathrm{mg} / \mathrm{dl}$ or creatinine clearance $40-60 \mathrm{~mL} / \mathrm{min}$. Patient performance status was $\leq 2$ according to WHO classification. Patients previously treated for a bladder tumor (recurrent bladder cancer).

Exclusion criteria: Immune compromised cases. Serum creatinine level $>2 \mathrm{mg} / \mathrm{dl}$. Inability to resect all visible intravesical tumor tissues. Follow up can't be done properly due to non-compliance, far residence or living lonely. Patient performance status $>2$.

Pre-operative evaluation: All patients were subjected to the following:

Thorough history taking with special attention to history of previous treatment for a bladder tumor and history of associated medical disease. Digital rectal examination (DRE) and bimanual examination were done. Standard laboratory investigations including complete blood picture, urine analysis and urine culture, renal and liver function tests, random, fasting and post prandial blood glucose level in known diabetic patients, and bleeding profile. Radiological investigations including pelvi-abdominal ultrasonography (US) combined with plain abdominal film (KUB), pelviabdominal computed tomography (CT) with and without contrast, bone scan and chest CT to rule out metastasis. Diagnostic urethro-cystoscopy and TUR biopsy (initial TUR) of all visible masses were done followed by histopathologic examination of the resected tissue with careful description of the findings.

\section{Outcomes and endpoints:}

Complete response (complete disappearance of all measurable lesions), partial response (more than $50 \%$ reduction of all measurable lesions or no increase in any lesion or no new lesions), stable disease (less than $50 \%$ reduction of measurable lesions or $<25 \%$ increase of measurable lesions), progression (which is defined as $>25 \%$ increase of any lesion, new lesion, recurrent tumor with higher stage or metastatic disease), and treatment failure which includes any of the following: partial response or stable disease after the end of treatment course, recurrence (which is defined as tumor identified at follow-up cystoscopy that was biopsied and fulgurated or resected previously with histopathological material showing urothelial malignancy), and progression. 


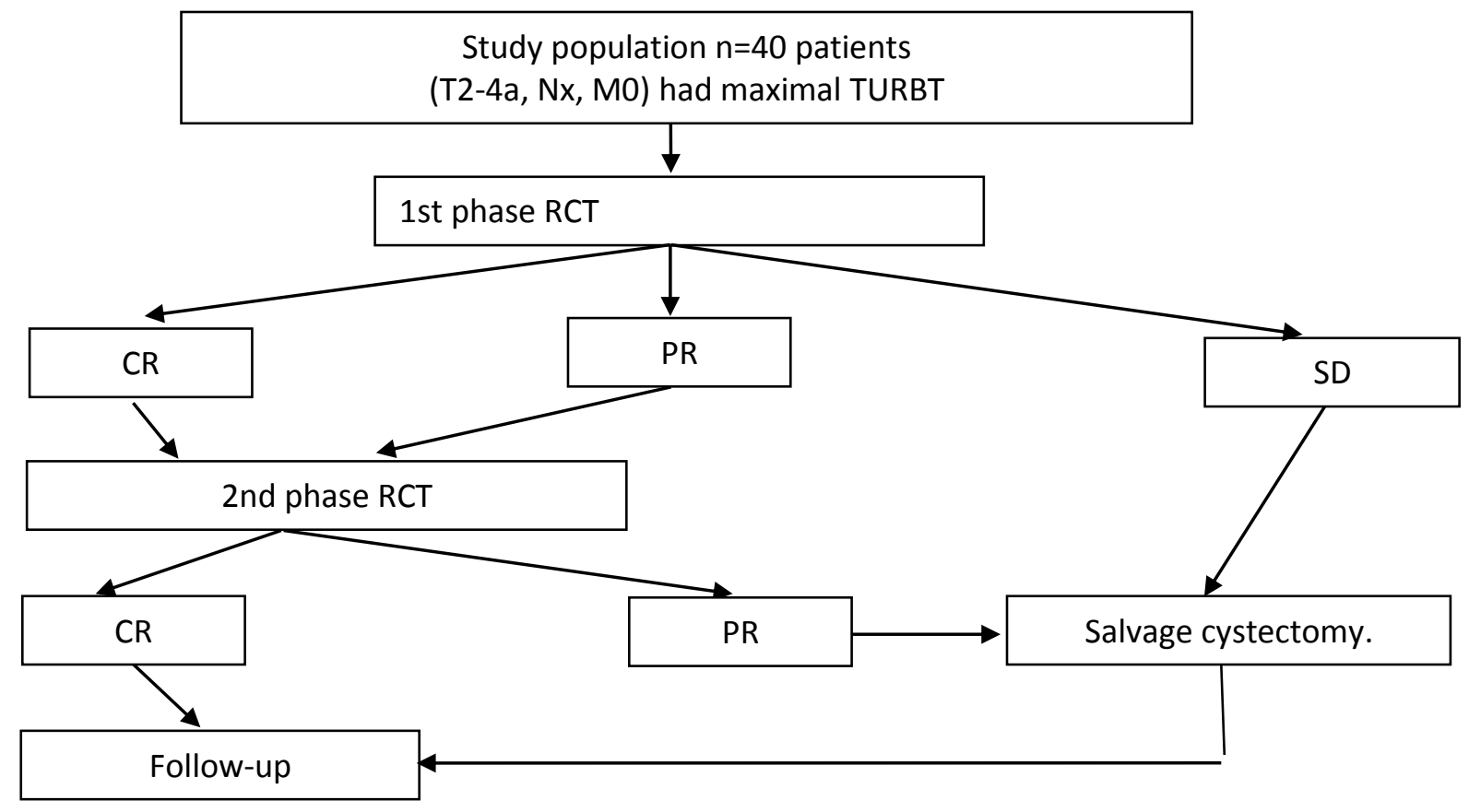

Scheme of treatment:

Figure 1: scheme of bladder preservation protocol.

Patients who achieved complete response or partial response in phase I were shifted to phase II treatment and patients who had stable disease were subjected to salvage cystectomy. After the end of treatment, patients who still had residual tumor underwent salvage cystectomy and patients who had complete response were monitored (figure 1).

\section{Treatment:}

The 40 patients who were eligible for the study were subjected to the following protocol: Maximal TURBT (which means removal of all visible intravesical tumors) with histopathologic examination of the resected tissue was done for all within 2 weeks from the initial TUR. Concurrent intra-venous gemcitabine infusion and radiotherapy were started within 2 weeks after maximal TURBT with a gemcitabine once weekly dose of $300 \mathrm{mg} / \mathrm{m}^{2}$ body surface area during radiotherapy course. In every patient, 60 Gy were given over 6 weeks using linear accelerator 4-6 mev as following: $1^{\text {st }}$ phase of total pelvic radiation consisted of 40 Gy divided into 5 fractions/week for 4 weeks with 4 gemcitabine injections. A lag time between the completion of the $1^{\text {st }}$ phase and the start of the $2^{\text {nd }}$ phase for 2 weeks during which follow up pelviabdominal CT and cystoscopy were done. According to response to the $1^{\text {st }}$ phase of treatment patients were decided to proceed to $2^{\text {nd }}$ phase or to proceed to salvage cystectomy. $2^{\text {nd }}$ phase of localized pelvic field dose consisted of 20 Gy divided into 5 fractions/week for 2 weeks with 2 gemcitabine injections. The sum of the radiotherapy course was 6 weeks.

Planning and simulation for radiotherapy were performed in the supine position with an empty bladder. Fixation with thermoplastic shells was used in obese patients and in patients having redundant abdomen. Contrast was given to visualize the bladder either intravenous or intravesically. Field arrangement for the proposed target volume was done by computerized planning system taking in consideration homogeneous distribution shape to the target volume and the tolerance dose to the critical organs. An isocentric four-field box method was used. The target field of radiotherapy in Phase I included the bladder, the proximal urethra and the pelvic lymph nodes. The treatment volume extended in the cranial-caudal dimension from S1-S2 interspace (mid-sacrum) to the lower pole of the obturator foramen (if bladder neck was involved: $1.5 \mathrm{~cm}$ below obturator foramen) and $1.5-2 \mathrm{~cm}$ beyond the pelvic bones laterally. The anterior border lies $1-2 \mathrm{~cm}$ in front of the anterior bladder wall and the posterior border at the mid-rectum (at 
least $1-3 \mathrm{~cm}$ posterior to the tumor). In phase II the field included the whole bladder with a margin of 2 $\mathrm{cm}$. Proper hydration and antiemetic (topisetron or ondanstron) were used before chemotherapy administration.

Follow-up:

All the patients were followed up according to our protocol at the end of phase II and quarter annually thereafter according to the following protocol: Follow-up visits included history taking and physical examination; urine analysis and cultures if were indicated, chest X-ray, and serum chemistries including liver and renal function tests. Follow-up cystoscopy every three months. Upper tract surveillance was performed by ultrasonography every six months and CT yearly. Quality of life was evaluated at the end of the study by NCCN-FACT FBISI18 (National Comprehensive Cancer Network-Functional
Assessment of Cancer Therapy-Functional Bladder Symptom Index) questionnaire.

Statistical analysis:

Differences in Prognostic factors affecting the response for treatment and the recurrence were calculated by Pearson's chi-square test with $p<$ 0.05 considered significant. Multivariate analyses were performed using logistic regression analysis for clinical response. Recurrence free and overall survival rates were calculated according to KaplanMeier actuarial method from the time of diagnosis. The contribution of each factor; multivariate analysis was performed by using the Cox proportional hazard regression model. Log rank test was used to compare survival rates. The statistical package used was XLSTAT Version 2013.4.08.

Results: The patient characteristics were shown in table 1 and the patients symptoms were shown in table 2 .

Table 1: Demographic characteristics

\section{Of the study population}

\begin{tabular}{lc}
\hline Number of patients & 40 \\
\hline Number of men/women & $32 / 8$ \\
\hline Median (range) age / years & $55(40-65)$ \\
\hline Mean age \pm SD/ years & $54.65 \pm 6.9$ \\
\hline Median (range) of follow-up time / months & $18(12-27)$ \\
\hline Mean follow-up time \pm SD/ years & $20 \pm 3.7$ \\
\hline
\end{tabular}

Table 2: Patient symptoms

\begin{tabular}{lcl}
\hline Symptom & No. & \% \\
\hline Hematuria & 32 & 80 \\
\hline Dysuria & 20 & 50 \\
\hline Frequency & 16 & 40 \\
\hline Necroturia & 14 & 35 \\
\hline Pyuria & 4 & 10 \\
\hline Suprapubic pain & 4 & 10 \\
\hline Dull aching loin pain & 2 & 5 \\
\hline Incontinence & 2 & 5 \\
\hline Clot retention & 2 & 5 \\
\hline
\end{tabular}

Tumor characteristics: In the initial radiological investigation, cystoscopic findings and pathology results: the lateral wall of the urinary bladder was the commonest site of involvement in 18 (45\%) patients. This is shown in table 3 .
Tumor response: At the end of phase I; CR was achieved in $22(55 \%)$ patients, PR in $8(20 \%)$ patients and SD in $10(25 \%)$ patients. Patients who had SD were subjected to salvage cystectomy. Patients who had CR and PR (30 patients) were 
enrolled in phase II. After phase II, of the 8 patients who had PR in phase I; 5 patients achieved CR and the remaining 3 patients underwent salvage cystectomy. At the end of treatment protocol, a sum of $27(67.5 \%)$ patients had CR with bladder preservation, while $13(32.5 \%)$ patients were subjected to salvage cystectomy (figure 2).

Table 3: Tumor characteristics

\begin{tabular}{|c|c|c|}
\hline Characteristics & No. & $\%$ \\
\hline $\begin{array}{l}\text { Tumor site : } \\
\text { Lateral wall } \\
\text { Posterior wall } \\
\text { Anterior wall } \\
\text { Dome } \\
\text { Base } \\
\text { Trigone }\end{array}$ & $\begin{array}{c}18 \\
9 \\
6 \\
5 \\
1 \\
1\end{array}$ & $\begin{array}{c}45 \\
22.5 \\
15 \\
12.5 \\
2.5 \\
2.5\end{array}$ \\
\hline $\begin{array}{l}\text { Tumor size : } \\
<5 \mathrm{~cm} \\
\geq 5 \mathrm{~cm}\end{array}$ & $\begin{array}{l}18 \\
22\end{array}$ & $\begin{array}{l}45 \\
55\end{array}$ \\
\hline $\begin{array}{l}\text { Concomitant CIS : } \\
\text { Present } \\
\text { Absent }\end{array}$ & $\begin{array}{c}2 \\
38 \\
\end{array}$ & $\begin{array}{c}5 \\
95 \\
\end{array}$ \\
\hline $\begin{array}{l}\text { Tumor grade : } \\
\text { Low } \\
\text { High }\end{array}$ & $\begin{array}{l}23 \\
17 \\
\end{array}$ & $\begin{array}{l}62.5 \\
37.5 \\
\end{array}$ \\
\hline $\begin{array}{l}\text { Bilharziasis : } \\
\text { Present } \\
\text { Absent }\end{array}$ & $\begin{array}{c}4 \\
36\end{array}$ & $\begin{array}{l}10 \\
90\end{array}$ \\
\hline
\end{tabular}

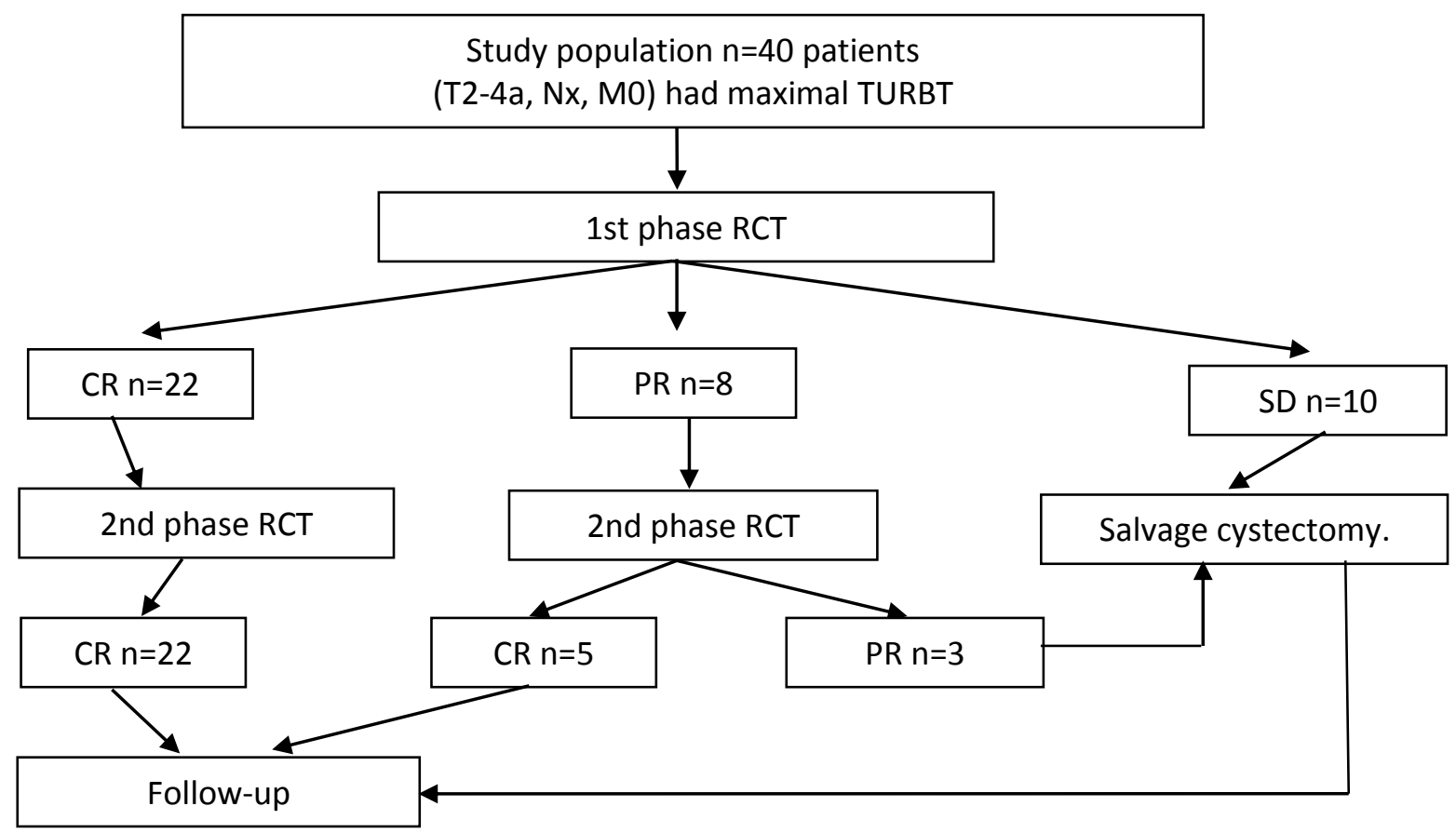

Figure 2: Treatment outcomes. 
Prognostic factors affecting the response of treatment: Table 4 shows the prognostic factors that might affect $\mathrm{CR}$ after phase I of radio-chemotherapy
(RCT). Stage, grade, obstructive uropathy, and concomitant CIS were better response (high CR rate).

Table 4: Prognostic factors after phase I of treatment.

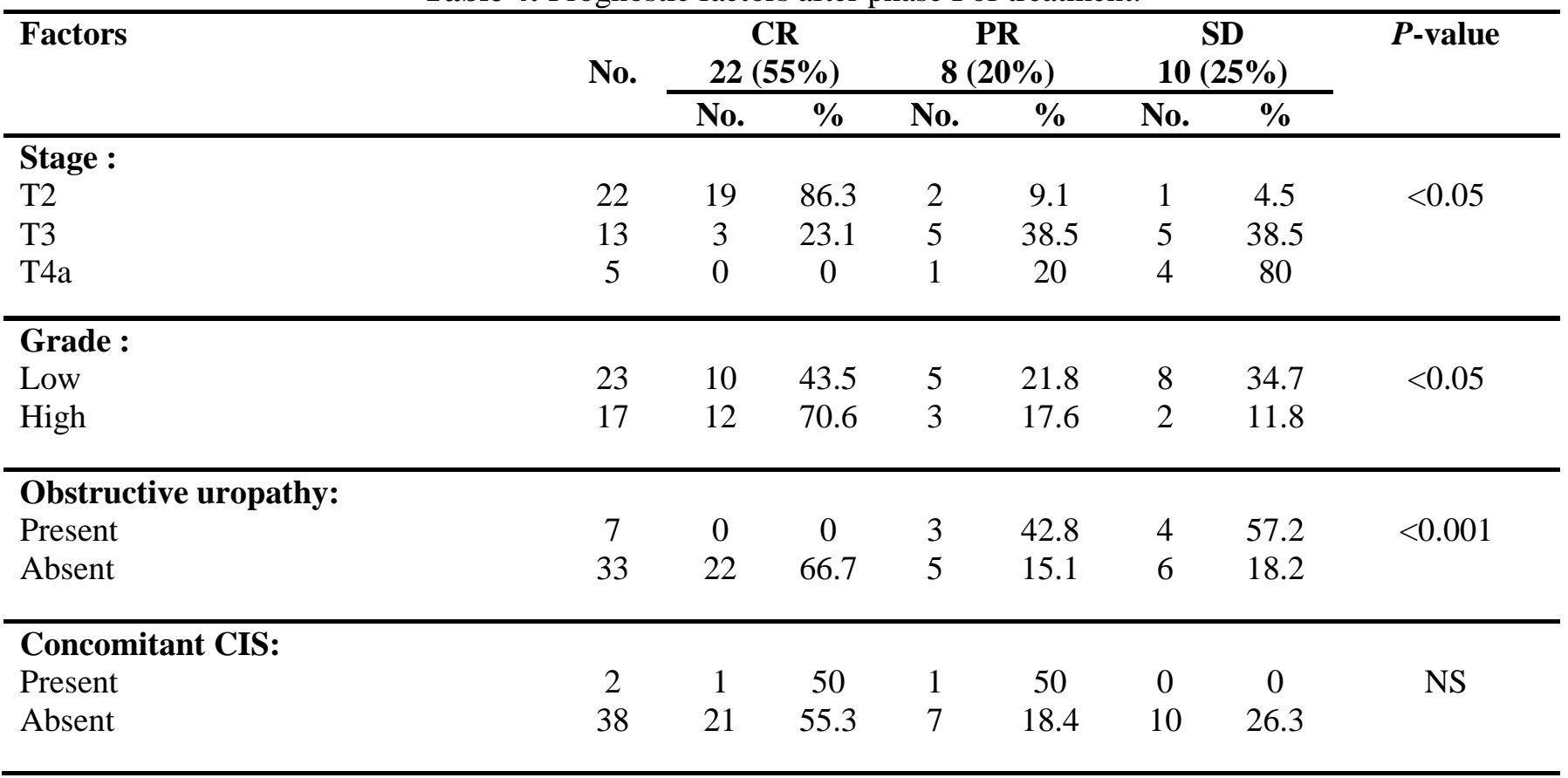

Table 5: Prognostic factors at the end of phase II.

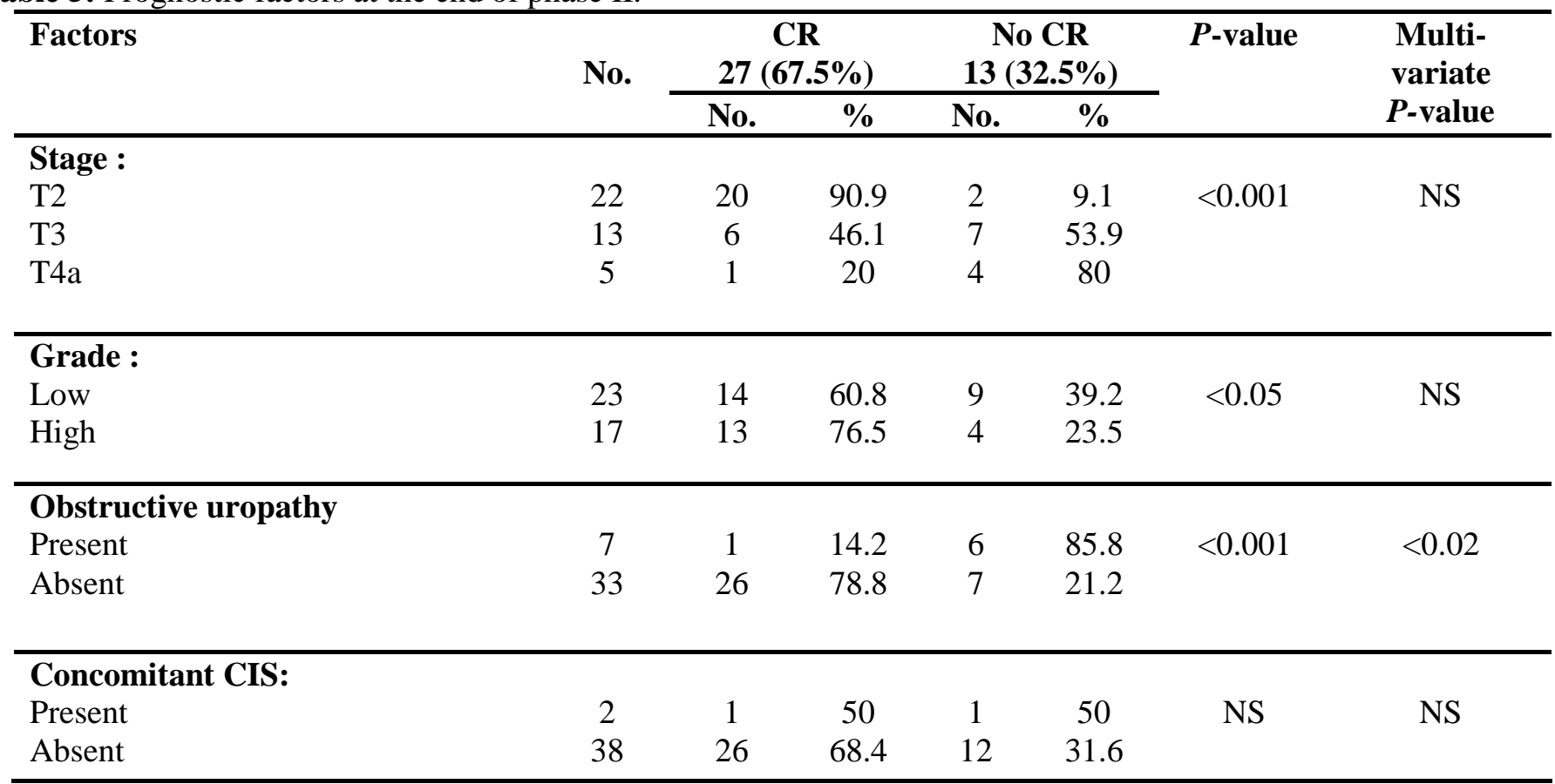

In addition, the same prognostic factors were studied after the end of treatment. It can be noticed that also early stages (T2), high grade, absence of obstructive uropathy were associated with high CR rate. In multivariate analyses obstructive uropathy remained significant prognostic factor affecting the response rate as shown in table 5.

Survival: The follow-up period present in this study ranged from 12 to 27 months with a median of 18 months. Two patients died during the study. One patient from myocardial infarction at 18 month and 
one from pulmonary embolism at 21 month (due to non-tumor cause). No patient developed distant metastasis during this period. The actuarial recurrence free survival (RFS) and overall survival were shown in figure 3,4. Stage, grade, concomitant CIS, obstructive uropathy, and the initial response of phase I were the factors studied in this study.
Table 6 shows that the stage of tumor and the absence of obstructive uropathy were the only significant factors $(p<0.001)$ affecting the relapse free survival. In multivariate analysis clinical $\mathrm{T}$ stage remained significantly associated with increased recurrence free survival (table 7).

Table 6: Factors affecting RFS of 27 patients TCC at end of study.

\begin{tabular}{|c|c|c|c|c|}
\hline Factors & No. & $\begin{array}{c}\text { RFS } \\
\text { n }(\%)\end{array}$ & $\overline{X^{2}}$ & $P$-value \\
\hline $\begin{array}{l}\text { Stage : } \\
\text { T2 } \\
\text { T3 } \\
\text { T4a }\end{array}$ & $\begin{array}{c}20 \\
6 \\
1\end{array}$ & $\begin{array}{c}20(100) \\
1(16.7) \\
0(0)\end{array}$ & 138.2 & $<0.001$ \\
\hline $\begin{array}{l}\text { Grade : } \\
\text { Low } \\
\text { High }\end{array}$ & $\begin{array}{l}14 \\
13 \\
\end{array}$ & $\begin{array}{l}11(78.5) \\
10(84.6)\end{array}$ & 0.2 & NS \\
\hline $\begin{array}{l}\text { Obstructive uropathy: } \\
\text { Present } \\
\text { Absent } \\
\end{array}$ & $\begin{array}{c}1 \\
26 \\
\end{array}$ & $\begin{array}{c}0(0) \\
21(80.7) \\
\end{array}$ & 74.3 & $<0.001$ \\
\hline $\begin{array}{l}\text { Concomitant CIS: } \\
\text { Present } \\
\text { Absent } \\
\end{array}$ & $\begin{array}{c}1 \\
26 \\
\end{array}$ & $\begin{array}{c}1(100) \\
25(96.2)\end{array}$ & 0.06 & NS \\
\hline $\begin{array}{l}\text { Initial response : } \\
\mathrm{CR} \\
\mathrm{PR}\end{array}$ & $\begin{array}{c}22 \\
5\end{array}$ & $\begin{array}{c}17(77.3) \\
4(80)\end{array}$ & 0.04 & NS \\
\hline
\end{tabular}

Table 7: The results of multivariate Cox proportional hazards analysis of prognostic variables of recurrence.

\begin{tabular}{lccc}
\hline & Hazards Ratio & $\boldsymbol{P}$-Value & 95\% Confidence interval \\
\hline Stage & 0.59 & $<0.001$ & $0.45-0.77$ \\
\hline Grade & 1.07 & 0.263 & $0.38-1.06$ \\
\hline Obstructive uropathy & 1.03 & 0.199 & $0.88-1.35$ \\
\hline Concomitant CIS & 1.63 & 0.491 & $0.52-6.37$ \\
\hline Initial response & 0.46 & 0.392 & $0.09-3.75$ \\
\hline
\end{tabular}




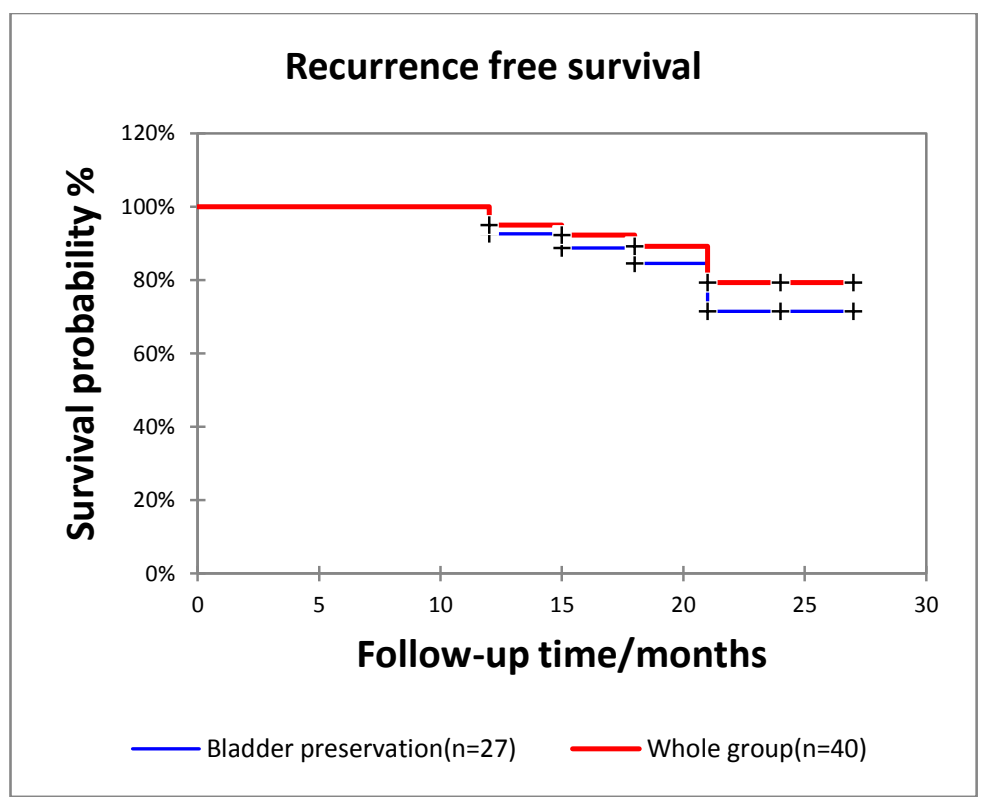

Figure 3: Kaplan-Meier analysis of recurrence-free survival of bladder preservation group and the whole group.

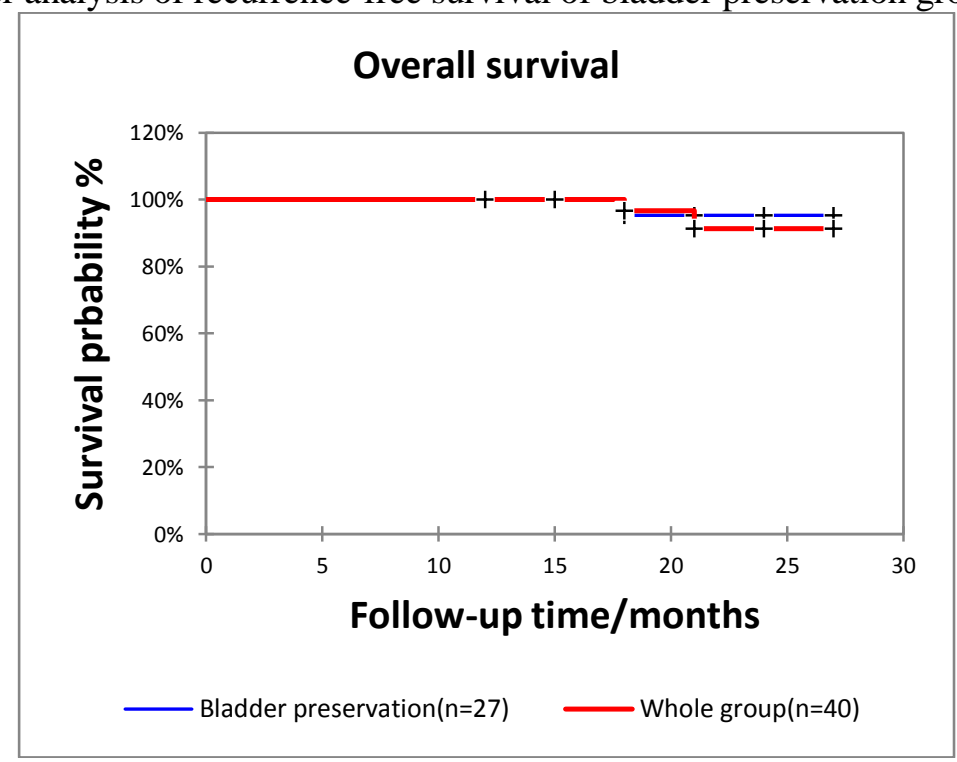

Figure 4: Kaplan-Meier analysis of overall survival of bladder preservation group and the whole group.

Recurrence: Of the 27 patients who had CR after phase II, 6 patients had tumor recurrence, 5 of them were muscle-invasive and 1 was non-muscle invasive recurrence. The non-invasive recurrence occurred at 12 months during follow-up and was treated with intravesical BCG. The invasive recurrences occurred at $12,15,18,21$ months during follow up and were treated with salvage cystectomy.

Treatment toxicity: All the 27 patients tolerated the treatment protocol and did not have life threatening events (grade III \& IV toxicity). Types toxicity encountered are listed in table 8 according to RTOG/EORTC (Radiation Therapy and Oncology Group and European Organization for Research into the Treatment of Cancer) criteria. Mild to moderate toxicity were observed and treated conservatively with supportive treatment without interruption of treatment course. 
Table 8: Toxicity grade of bladder preservation group.

\begin{tabular}{lccc}
\hline Toxicity & \multicolumn{3}{c}{ Grade of toxicity } \\
\cline { 2 - 4 } & $\mathbf{0}$ & I & II \\
\hline Skin erythema or desquamation & $9(33.3 \%)$ & $15(55.6 \%)$ & $3(11.1 \%)$ \\
\hline Anaemia & $18(66.7 \%)$ & $9(33.3 \%)$ & 0 \\
\hline Leucopenia & $22(81.5 \%)$ & $5(18.5 \%)$ & 0 \\
\hline Diarrhea & $10(37 \%)$ & $15(55.6 \%)$ & $2(7.4 \%)$ \\
\hline Nausea & $5(18.5 \%)$ & $12(44.5 \%)$ & $10(37 \%)$ \\
\hline Vomiting & $12(44.5 \%)$ & $10(37 \%)$ & $5(18.5 \%)$ \\
\hline Dysuria & $1(3.8 \%)$ & $10(37 \%)$ & $16(59.2 \%)$ \\
\hline Frequency & 0 & $10(37 \%)$ & $17(63 \%)$ \\
\hline Creatinine elevation & $23(85.2 \%)$ & $4(14.8 \%)$ & 0 \\
\hline
\end{tabular}

Quality of life: After subjecting the patients to NCCNFACT FBISI18 (National Comprehensive Cancer Network-Functional Assessment of Cancer Therapy-

Table 9: QOL of bladder preservation group.
Functional Bladder Symptom Index) questionnaire, the mean values of quality of life score were shown in table 9.

\section{Questionnaire item $\quad$ Mean \pm SD}

value

\begin{tabular}{|c|c|c|}
\hline \multirow[t]{9}{*}{ Physical disease related symptoms subscale } & Pain & $3.1 \pm 0.8$ \\
\hline & Weight loss & $3.4 \pm 0.5$ \\
\hline & Trouble in urine control & $2.8 \pm 0.9$ \\
\hline & General Weakness & $2.7 \pm 0.9$ \\
\hline & Dizziness & $3 \pm 1$ \\
\hline & Trouble in meeting family needs & $2.8 \pm 0.8$ \\
\hline & Appetite & $3.2 \pm 0.7$ \\
\hline & Erection (men only) & $2.9 \pm 0.8$ \\
\hline & Good sleeping & $2.8 \pm 0.8$ \\
\hline \multirow[t]{2}{*}{ Emotional disease related symptoms subscale } & Worry about the illness & $2.4 \pm 1.1$ \\
\hline & Sadness & $2.1 \pm 1$ \\
\hline \multirow[t]{5}{*}{ Treatment side effects subscale } & Nausea & $3.3 \pm 0.7$ \\
\hline & Lack of energy & $2.9 \pm 1$ \\
\hline & Feeling ill & $2.5 \pm 1.2$ \\
\hline & Bowel control & $3.2 \pm 1$ \\
\hline & Bothering from side effects & $3.1 \pm 0.9$ \\
\hline \multirow[t]{2}{*}{ Function and well being subscale } & Enjoying life & $2 \pm 0.9$ \\
\hline & Satisfaction with quality of life & $2.4 \pm 1.1$ \\
\hline Total & & $50.6 \pm 16.1$ \\
\hline
\end{tabular}

\section{DISCUSSION}

Muscle-invasive bladder cancer is a common malignancy with a high mortality rate. Despite ongoing debates about the optimal primary intervention, radical cystectomy remains the cornerstone of first-line therapy in many institutions. Over the past decade, bladderpreserving strategies involving transurethral resection (TUR), chemotherapy and radiotherapy have evolved. (1).
Supporters of bladder preservation depend on First, the comparable response rates, recurrence and progression free survival rates, and overall survival with radical cystectomy. Second, the better quality of life and the tolerable treatment course. While opposers say that, when comparing studies of each approach, there are two main caveats. First, surgical series involve pathologically staged tumors as opposed to clinically staged tumors in the chemoradiation trials, favoring the surgical series because many cancers are upstaged at the 
time of surgery. Second, retrospective radical cystectomy series do not report an intent-to-treat analysis, which is in contrast to prospective chemoradiation trials. Randomized trials comparing these two treatments are unlikely to be forthcoming in the near future and are based, in part, on physician biases. (2).

In the present study, patients were treated with combined chemo-radiotherapy. After phase I, complete response (CR) was $55 \%$ and partial response (PR) was 20\%. After phase II complete response (CR) was $67.5 \%$.

These results are similar to (Peter W.M. et al., 2007) (3) in a large study done on 340 patients. Two hundred and forty seven patients received radiotherapy alone, 36 received radiotherapy and concurrent chemotherapy, and 57 received neoadjuvant chemotherapy followed by radiotherapy. Overall complete response was obtained in $63.5 \%$ of patients, and median follow-up was 7.9 years.

(George L, et al., 2004) (4) found that 45 out of 60 patients receiving concomitant chemo-radiotherapy showed CR (75\%) and (Rincon A, et al., 2010) (5) found $(72 \%) \mathrm{CR}$.

In the Massachusetts General Hospital series of 190 patients treated by trimodality therapy between 1986 and 1998, 63\% exhibited a CR (6) .

In another series from Germany and Spain, CR was $80 \%$ and $89 \%$ respectively (7). The RTOG trials demonstrated a CR rate after induction in $75 \%$ and $59 \%$ of the patients (6). It is also similar to other Egyptian results. (Hanan Shawky, 2007) (8) and RTOG twice daily protocol revealed $81 \%$ CR after induction phase. (Kaufman DS, et al., 2009)(9). This may be due to good performance status with normal renal, hepatic, and blood picture, early stage (T2), absence of obstructive uropathy, and complete resection of the tumor by TUR.

In the current study, 22 of $27(81.5 \%)$ patients maintained their bladder without recurrence. Our results regarding recurrence are within the range of the reported results (14\%-20\%) (10).

Survival analysis was performed as an initial result for recurrence free survival (RFS) and overall survival. For all patients, the recurrence free survival (RFS) and overall survival in the first year of follow up were $100 \%$. At 18 months, RFS was $84 \%$ while overall survival was $100 \%$. At the end of the study, RFS was $72 \%$ while overall survival was $96 \%$.

These results are higher than those of (Rodel, et al., 2002) (10), who reported $74 \%$ RFS and $80 \%$ overall survival at 18 months. This may be due to different modality of treatment, where, 289 patients in Rodel's study were treated by RCT and 126 were treated by RT alone. On the other hand (Arias F, et al., 2000)(11), reported $82 \%$ RFS and $90 \%$ overall survival at 18 months.

However, these results are lower than (Aboziada $M$, et al., 2009)(12), who reported $88.5 \%$ RFS and $100 \%$ overall survival at 18 months.

In a large comparative study done by (Ott $\mathrm{OJ}$, $\boldsymbol{e t}$ al. ,2009) (13) Contemporary radical cystectomy series reported 5-year overall survival rates of $63-74 \%$ for superficial and $26-63 \%$ for muscle-invasive disease while bladder preservation protocols achieved 48-52\% 5-year overall survival rate for superficial and $34-50 \%$ for muscle-invasive disease.

In appropriately selected patients, bladder preserving treatment with complete transurethral resection, radiation therapy, and concurrent chemotherapy offers a probability of long-term cure and overall 5-year survival comparable to cystectomy-based approaches (49\% to $63 \%$ ) in patients of similar clinical stage and age. The 5-year survival rate with bladder preservation ranged between $38 \%$ and $45 \%$. In addition, these bladder-preserving approaches result in approximately $80 \%$ of the long-term survivors maintaining a normal functioning bladder (14).

Results of bladder sparing approach need to be compared with standard cystectomy. Unfortunately, primary cystectomy has not yet been tested against combined-modality bladder sparing treatment in randomized trials. In a published series of 1,054 patients with bladder cancer treated between 1971 and 1997 with radical cystectomy, (Stein JP, et al., 2001) (15) reported excellent overall survival rates that should be considered as the surgical standard to which other treatment modalities should be compared. They reported a 5-year overall survival of $47 \%$ for the group of invasive bladder cancer. This figure is comparable to the results of RTOG trials 2,12,16 in which 5-year survival rates of $50 \%-60 \%$, and 5-year survival rates with an intact bladder of $40 \%-45 \%$ for patients treated with bladder sparing approach were reported. It seems that the present approach in treating bladder cancer patients with TUR and chemo-radiotherapy is a reasonable one alternative to classic surgical approach in selected patients (14).

However, larger follow-up and a large number of patients are needed to validate these results of RTOG trials. For patients who have undergone cystectomy for invasive TCC of the bladder, (Shoji, et al., 2001) (16) reported 18 months actuarial overall survival of $90 \%$ for 
pT2 and 50\% for pT3. For patients without lymph node metastasis and patients with lymph node metastasis, the OS was $85 \%$ and $42 \%$, respectively. (Gaitonde, et al., 2002) (17) reported 18 months actuarial overall survival 94\% for pT2-pT3a without lymph node metastasis and 92\% for all pT2-pT3a patients. These results are slightly lower than ours where the 18 months actuarial overall survival was $100 \%$ for all patients.

This study showed that early stage (T2), high grade, absence of obstructive uropathy and complete TUR were associated with better response (high CR rate). While (Rodel, et al., 2002) (10) found that significant factors affecting CR after completion of radio-chemotherapy (RCT) were disease stage and extent of TUR. However, (Sauer, et al., 1998) (18) found that disease stage, histological grade, and extent of TUR were the only significant factors. The presence of hydronephrosis at the time of diagnosis also impacts treatment success. In the other series (6) , 27 of 190 patients had hydronephrosis initially, and their complete response rate was $37 \%$, compared to $68 \%$ in patients without hydronephrosis. (Aboziada M, et al., 2009) (12) found that early stage (T2), high grade, absence of bilharziasis, absence of obstructive uropathy and complete TUR were associated with better response.

Another major argument against the use of bladder conservation therapy has been the potential long-term effects of radiation on bladder function. The Massachusetts General Hospital (MGH) group has performed QOL and urodynamic studies (UDS) in 71 patients who are alive with a functioning bladder. The median time from trimodality treatment was 6.3 years, long enough for late effects to arise. Of interest, $75 \%$ of patients had normally functioning bladders by UDS. Reduced bladder capacity was identified in $22 \%$ of patients, and only in a third of these patients did distressing symptoms arise. Bowel symptoms occurred in $22 \%$ of patients with $14 \%$ recording any level of distress. Only $8 \%$ of patients reported dissatisfaction with their sex lives. In contrast, in the Swedish and Italian series, $13 \%$ and $8 \%$, respectively, of cystectomised controls retained useful erection (14).

In our study, all patients completed the treatment protocol with tolerable toxicity profile and did not have grade III and IV of toxicity. Although pelvic irradiation and concomitant gemcitabine may locally eradicate the cancer, there are recognized hematological, bowel and urinary consequences.

This study showed that in spite of a relatively small number of patients and short follow up period; selective bladder preservation using the combination of complete transurethral resection of tumor, IV gemcitabine and radiotherapy is a good chance of the patients to achieve a high complete response rate in the treatment of invasive TCC of the bladder with better quality of life.

\section{REFERENCES}

(1) Sherwood B. T., Jones, J. K. Mellon G. D. D., Kockelbergh R. C., Steward W. P., Symonds R. P. (2005) : Concomitant Chemoradiotherapy for Muscleinvasive Bladder Cancer: The Way Forward for Bladder Preservation? The Royal College of Radiologists. J Clinical oncology. Published by Elsevier 2005; 160166.

(2) Mutahir A, Mushabbab Al Asiri, Mohsin Fareed, et al. (2012) : Bladder Preservation with Concurrent Radiotherapy and Gemcitabine following Maximal Transurethral Resection for Muscle Invasive Bladder Cancer: Single Institutional Experience. J Nucl Med Radiat Ther 3:1-5.

(3) Peter W.M.,et al. (2007) : Long-term outcome of radiation-based conservation therapy for invasive bladder cancer. Urologic Oncology: Seminars and Original Investigations 25 (2007) 303-309.

(4) George L, Bladou F, Bardou VJ, et al. (2004) : Clinical outcome in patients with locally advanced bladder carcinoma treated with conservative multimodality therapy. Urology 2004; 64(3): 488-93.

(5) Rincón A. Mayans, D. Rosell Costa, J.J. Zudaire Bergera, et al. (2010): Response and progression-free survival in T2 to T4 bladder tumors treated with trimodality therapy with bladder preservation: Spainactas urol esp. 2010; 34(9): 775-780.

(6) Shipley WU, Kaufman DS, Zehr E, et al. (2002) : Selective bladder preservation by combined modality protocol treatment: long-term outcomes of 190 patients with invasive bladder cancer. Urology. 2002, 60: 62-7.

(7) Almudena Zapatero, Carmen Martin de Vidales, Ramón Arellano, et al. (2009) : Updated results of bladder sparing trimodality approach for invasive bladder cancer. Urologic Oncology: Seminars and Original Investigations (2009).

(8) Hanan Shawky Gamal el-Deen. (2007) : Initial Results of Retrospective Study: Preoperative Transurethral Excision Plus Chemotherapy and Radiation Therapy and Trial of Bladder Preservation. Journal of the Egyptian Nat. Cancer Inst., Vol. 19, No. 2, June. 2007, 133- 146.

(9) Kaufman DS, Winter KA, Shipley WU, et al. (2009) : Phase I-II RTOG Study (99-06) of Patients With Muscle- Invasive Bladder Cancer Undergoing Transurethral Surgery, Paclitaxel, Cisplatin, and Twicedaily Radiotherapy Followed by Selective Bladder Preservation or Radical Cystectomy and Adjuvant Chemotherapy. UROLOGY. 2009, 73: 833-837. 
(10) Rodel C, Grabenbauer GG, Kuhn R, et al. (2002) : Organ preservation in patients with invasive bladder cancer: initial results of an intensified protocol of transurethral surgery and radiation therapy plus concurrent cisplatin and 5-fluorouracil. Int J Radiat Oncol Biol Phys. 2002, 52: 1303-9.

(11) Arias F, Dominguez MA, Martinez E, et al. (2000) : Chemo-radiotherapy for muscle invading bladder carcinoma. Final report of a single institutional organsparing program. Int J Radiat Oncol Biol Phys. 2000, 47: $373-8$

(12) Aboziada M, et al., (2009) : Initial Results of Bladder Preserving Approach by Chemo-Radiotherapy in Patients with Muscle Invading Transitional Cell Carcinoma. Journal of the Egyptian Nat. Cancer Inst., Vol. 21, No. 2, June: 167-174, 2009.

(13) Ott OJ, et al. (2009) : Radiochemotherapy for Bladder Cancer. Clinical Oncology (2009) 21: 557-565.

(14) Michaelson D, Zietman A. (2004) : Invasive bladder cancer: the role of bladder-preserving therapy. In: American Society of Clinical Oncology. 2004 educational book. Proceedings of the 40th ASCO
Annual Meeting; 2004 June 5-8; New Orleans, LA. p.457-65.

(15) Stein JP, Lieskovsky G, Cote R, et al. (2001) : Radical cystectomy in the treatment of invasive bladder cancer: Long-term results in 1,054 patients. J Clin Oncol. 2001, 19: 666-675.

(16) Shoji H, Hideaki M, Masato F, et al. (2001) : Prognostic variables in patients who have undergone radical cystectomy for TCC of the bladder. Jpn J Clin Oncol. 2001, 31, 399-402.

(17) Gaitonde K, Goyal A, Nagaonkar S, et al. (2002) : Retrospective review and long-term follow up of radical cystectomy in a developing country. BJU International. 2002, 89: 57-61.

(18) Sauer R, Birkenhake S, Kuhn R, et al. (1998) : Efficacy of radio-chemotherapy with platin derivatives compared to radiotherapy alone in organ-sparing treatment of bladder cancer. Int J Radiat Oncol Biol Phys. 1998, 40: 121-127. 